\title{
Control Strategy of Parallel Systems with Efficiency Optimisation in Switched Reluctance Generators
}

Research Article

\author{
Xiaoshu Zan ${ }^{1,2}$, Hang Lin ${ }^{2, *}$, Guanqun Xu², Tiejun Zhao², Yi Gong² \\ ${ }^{\top}$ State Key Laboratory of Operation and Control of Renewable Energy \& Storage Systems (China Electric Power Research Institute), Beijing, China \\ ${ }^{2}$ School of Electrical and Power Engineering, China University of Mining and Technology, Xuzhou, China
}

Received: March 27, 2021; Accepted: May 04, 2021

\begin{abstract}
To solve motor heating and life shortening of parallel switched reluctance generator (SRG) induced by uneven output currents due to different external characteristics, we generally adopt current sharing control (CSC) to make each parallel generator undertake large load currents on average to improve the reliability of parallel power generation system. However, the method usually causes additional loss of power because it does not consider the efficiency characteristics of each parallel generator. Therefore, with the efficiency expression for the parallel system of SRG established and analysed, the control strategy based on differential evolution (DE) algorithm is proposed as a mechanism by which to enhance generating capacity and reliability of multi-machine power generation from the perspective of efficiency optimisation. We re-adjust the reference current of each parallel generator to transform the working point of each generator and implement the efficiency optimisation of parallel system. The performance of the proposed control method is evaluated in detail by the simulation and experiment, and comparison with traditional CSC is carried out as well.
\end{abstract}

Keywords: switched reluctance generator • parallel system • efficiency optimization • differential evolution algorithm

\section{Introduction}

Switched reluctance generator (SRG), a kind of the special motor free of rare-earth materials, possesses the advantages of both simple structure and flexible switching between electric and power states $(\mathrm{Li}$ and $\mathrm{Li}$, 2002; Torrey, 2002). Compared with three-stage brushless synchronous starter/generator system (Oyama et al., 2010), electromagnetic doubly salient starter/generator system (Zhang et al., 2018) and asynchronous motor starter/generator system (Hu et al., 2006), switched reluctance starter/generator system (Cai and Deng, 2012; Chen and $\mathrm{Gu}, 2010$ ) can reach the demand of supply system in many fields, such as electric vehicles, wind power generation and aerospace engineering (Barros et al., 2017; Gan et al., 2017; Krishnamurthy et al., 2006). Despite the availability of many advantages, the power generation capacity of single-machine is not sufficient. Therefore, experts and scholars at home and abroad have been devoted to the question of how to enhance the efficiency of parallel power generation system (Liu et al., 2002; Peng et al., 2011; Zhang and Pan, 2004).

To enhance the reliability, anti-interference ability and generation capacity of power generation system (Chang and Liaw, 2008; Sun et al., 2011; Xiong et al., 2009), SRGs are connected in parallel on the basis of improving the generation capacity of a single SRG so as to fulfil the actual demand of various loads. However, the generator overload and uneven power distribution caused by different external characteristics of each generator usually make performance of parallel system to undergo degradation (Chen et al., 2014; Zhou et al., 2019). Therefore, addressing the question of how to rationally allocate power generation between each SRG is the key to design a parallel control strategy (Panov et al., 2008; Schofield et al., 2009; Song et al., 2014). 
Current sharing control (CSC), a general method, is used to keep the output power equilibrium between each parallel SRG (Choi et al., 2014; Rahmanian et al., 2017; Sikder et al., 2014). Although the traditional CSC can implement effective distribution of load power, it cannot realise the optimisation of system efficiency.

According to the operation of parallel SRGs, Ju (2008) pointed out that external CSC chip was used to implement sharing of current but it reduced the reliability of the whole system to a certain extent. Zhou (2011) proposed that CAN bus communication is combined with traditional CSC. The method made up for the shortcoming of masterslave CSC method. Ren et al. (2019) proposed a CSC method based on virtual impedance to aim at the LLC resonant converter in parallel. The method implemented easy current sharing and extended modules. Du et al. (2019) proposed, compared with the traditional CSC, a finite time control method for the CSC of DC-DC converters to enhance the responding speed and steady-state performance. Chen et al. (2016) proposed that the proportional integral sliding mode control can be combined with master-slave CSC method to enhance the robustness of DC-DC parallel system with the capacity of current sharing. Based on the analysis of the above methods, it is found that an effective control strategy for parallel operation of SRGs is essential. Therefore, according to actual operation condition, we can re-adjust the output currents of each parallel SRG to implement the optimisation of overall efficiency and reduce energy losses.

In this paper, an uneven current distribution control method is proposed to implement efficiency optimisation of parallel power generation system. We take the general efficiency of parallel power generation system as the optimal objective. To solve the nonlinear problem of SRG, differential evolution (DE) algorithm is introduced to optimise currents of each phase winding. The rest of this paper is organised as follows. In Sections $2-4$, the efficiency optimisation model of parallel power generation system is established on the basis of the calculation formula of general efficiency and the expression of efficiency characteristics of single SRG. Section 5 describes the optimisation process based on DE algorithm and designs the key parameters of algorithm. In Section 6, the output voltage and current waveforms are simulated under the traditional CSC method and the efficiency optimisation control (EOC) method. In Section 7, experiments of uneven current distribution scheme are carried out. The feasibility and effectiveness of the proposed control method are verified by comparing the actual efficiency of different control methods under different load currents.

\section{Efficiency Characteristics of SRG}

We first analyse the energy distribution of a single SRG to implement the optimisation of multi-parallel power generation system. Figure 1 shows the energy flow diagram of a single SRG, with less mechanical loss ignored. Therefore, we can obtain the efficiency of a single generator, as shown in Eq. (1). $T_{m}$ and $T_{e}$ are the mechanical torque and electromagnetic torque of SRG, respectively, whereas $U_{L}$ and $I_{\text {out }}$ are the load voltage and output current of SRG, respectively, in Eq. (1).

It can be found from Eq. (1) that the efficiency characteristic of SRG is approximately regarded as a function of the efficiency and output current when the input power is constant and the output voltage is stable at the rated value. Therefore, the efficiency of SRG under different load current is recorded through transforming the load resistance of output port of SRG. The efficiency characteristics curves of two parallel SRGs are obtained by quintic polynomial fitting.

$$
\eta=\frac{P_{2}}{P_{1}}=\frac{U_{L} I_{\text {out }}}{T_{m} \omega} \approx \frac{P_{2}}{P_{e}}=\frac{U_{L} I_{\text {out }}}{T_{e} \omega}
$$

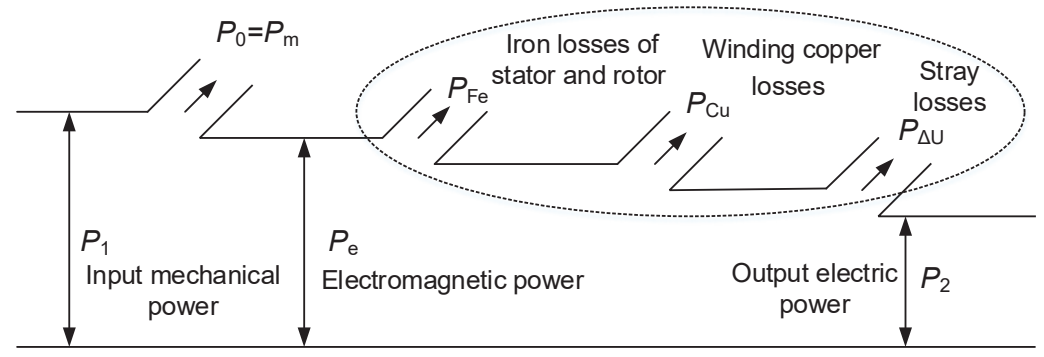

Fig. 1. Energy flow diagram of SRG. SRG, switched reluctance generator. 


\section{Design Scheme with Efficiency Optimisation of Parallel System}

The overall efficiency of parallel power generation system with M SRGs is

$$
H=\frac{P_{\text {out }}}{P_{\text {in }}}=\frac{U_{L} I_{L}}{\sum_{k=1}^{M} \frac{U_{L} I_{L}}{\eta_{k}}}=\frac{I_{L}}{\sum_{k=1}^{M} \frac{I_{k}}{\eta_{k}}}
$$

where $H$ is the overall efficiency of parallel power generation system; $P_{\text {out }}$ and $P_{\text {in }}$ are the overall output power and input power of system, respectively; $M$ is the number of parallel generators; $I_{k}$ and $I_{L}$ are the output current of the $K$ th generator and the load current, respectively; and $\eta_{k}$ is the efficiency of the Kth generator.

Equation (2) shows that $H$ reaches the maximum when the load is constant and the denominator reaches the minimum. Therefore, the efficiency optimisation problem of parallel power generation system can be converted into the following conditional extremum problem.

$$
\left\{\begin{array}{l}
\max (H)=\frac{I_{L}}{\min \left(\sum_{k=1}^{M} I_{k} / \eta_{k}\right)} \\
I_{M I N(k)} \leq I_{k} \leq I_{M A X(k)} \\
\sum_{k=1}^{M} I_{k}=I_{L}
\end{array}\right.
$$

$I_{\text {MIN(k) }}$ and $I_{\text {MAX(k) }}$ are the minimum output current and maximum output current of the Kth generator in Eq. (3). To solve the above conditional extremum problem, the condition extremum is generally converted into unconditional extremum with constraints. Therefore, Eq. (3) can be further transformed into:

$$
\left\{\begin{array}{c}
\max (H)=\frac{I_{L}}{\min \left(\sum_{k=1}^{M-1} \frac{I_{k}}{\eta_{k}}+\frac{I_{L}-\sum_{k=1}^{M-1} I_{k}}{\eta_{M}}\right) \quad(\quad(k=1, \ldots, M-1)} \\
I_{M I N(k)} \leq I_{k} \leq I_{M A X(k)}, I_{M I N(M)} \leq I_{L}-\sum_{k=1}^{M-1} I_{k} \leq I_{M A X(M)}
\end{array}\right.
$$

To obtain the extremum of multivariate function, the partial derivatives of $\left(I_{1}, I_{2}, \ldots, I_{M-1}\right)$ are calculated, respectively, and then the stagnation points of function $\left(I_{1}, I_{2}, \ldots, I_{M-1}\right)$ are obtained; and afterwards the answer is verified with sufficient conditions. However, the above method must satisfy the prerequisite condition with existing partial derivative. In addition, there are many problems in practice, such as a large amount of calculation and slow convergence speed. Therefore, the DE algorithm is used for optimisation to obtain stronger global convergence ability and inherent parallelism. Compared with other optimisation algorithms, DE algorithm can implement massive parallel distributed processing and bring about a decline in the optimisation time.

Figure 2 shows that the output current distribution scheme of parallel SRGs under efficiency optimisation. According to the principle of efficiency optimisation, the new current allocation algorithm is introduced into the traditional CSC in the scheme. We adopt the algorithm to obtain the reference current of each SRG. The adjustment of the reference current makes the output current redistribution to optimise the overall efficiency of system.

The efficiency can be talked only in the system at steady-state, and thus efficiency optimisation under the transient condition will affect the security and reliability of system. Therefore, the CSC method is generally used to maintain stability of the system in the transient condition during actual operation. The EOC method is selected under system steady-state to implement the optimisation of the overall efficiency. 


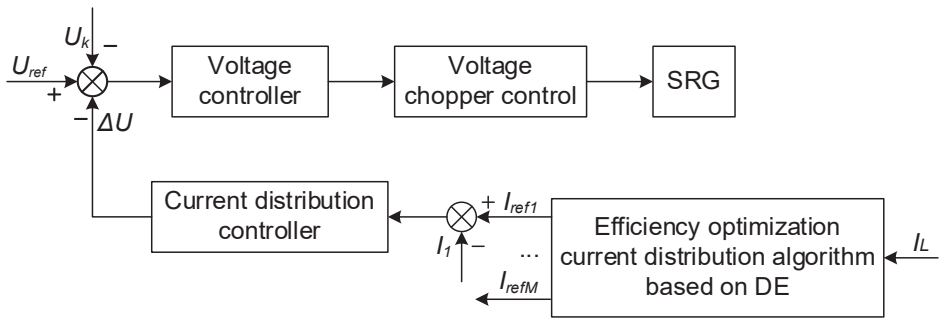

Fig. 2. Current distribution control method based on efficiency optimisation. DE, differential evolution; SRG, switched reluctance generator.

\section{Angle Optimisation of a Single SRG}

Both the turn-on angle $\left(\theta_{o n}\right)$ and the turn-off angle $\left(\theta_{\text {off }}\right)$ have an effect on power generation efficiency. The $\theta_{\text {on }}$ and $\theta_{\text {off }}$ must be optimised to minimise the energy losses of a single SRG.

The variations between the extinction angle and the generation efficiency under different rotating speeds are shown in Figure 3. The load resistance takes $5 \Omega$, and the $\theta_{\text {on }}$ is set at the range of $20-28^{\circ}$ with $2^{\circ}$ as the interval.

Figure 3 shows that the efficiency increases at first and then decreases with the increase of extinction angle when the rotating speed is constant and the $\theta_{\text {off }}$ changes. The power generation efficiency under different $\theta_{\text {on }}$ essentially maintains its consistent nature till $\theta_{\text {off }}$ increases to a certain value. The maximum efficiency points of power generation move backwards with the increase of rotating speed. Each phase conduction interval gradually becomes wider. Table 1 shows the $\theta_{\text {on }}$ and $\theta_{\text {off }}$ with the maximum efficiency under different speeds.
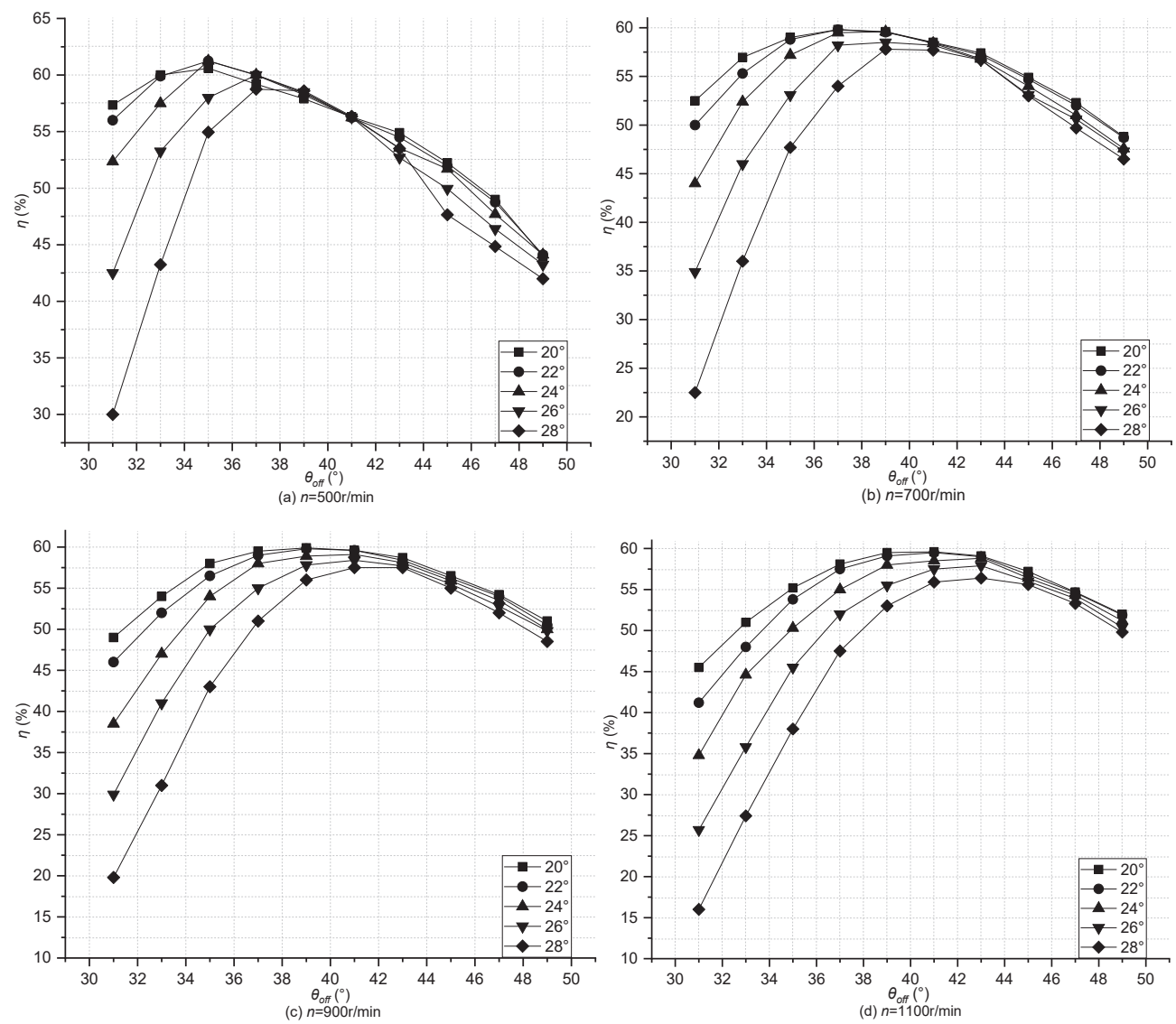

Fig. 3. Continued 

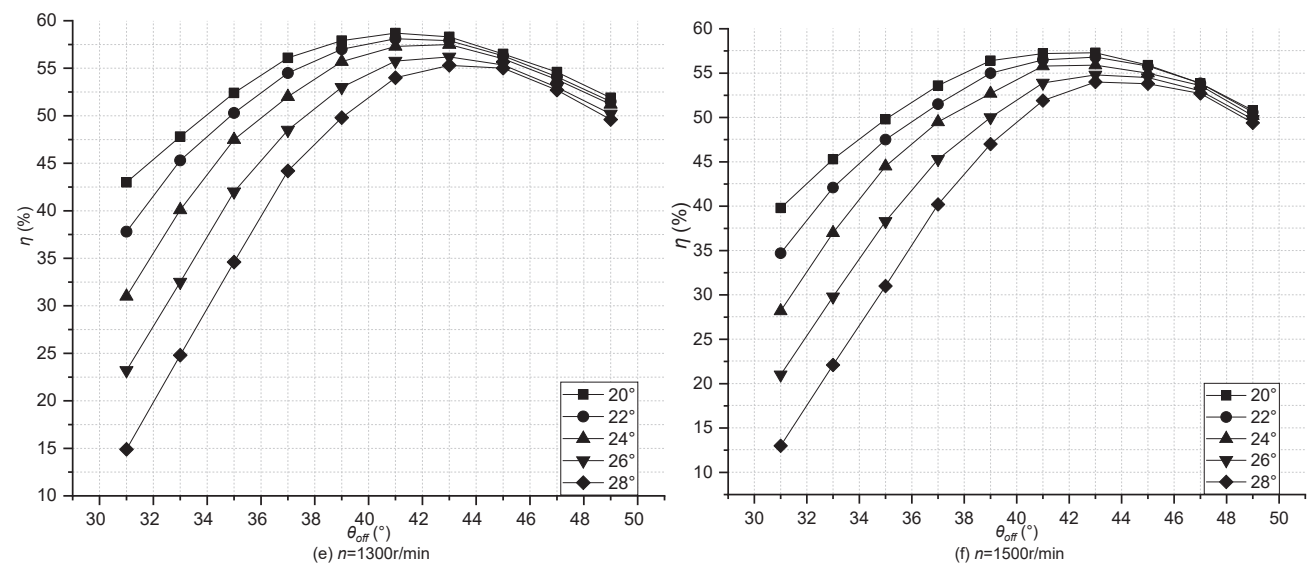

Fig. 3. The variation relation between efficiency, $\theta_{\text {on }}$ and $\theta_{\text {off }}$ under different speeds.

Table 1. The optimal $\theta_{\text {on }}$ and $\theta_{\text {off }}$ under different rotating speeds

\begin{tabular}{lcc}
\hline Speed $(\mathrm{r} / \mathrm{min})$ & $\theta_{\text {on }}\left({ }^{\circ}\right)$ & $\theta_{\text {off }}\left({ }^{\circ}\right)$ \\
\hline \hline 500 & 22 & 35 \\
700 & 22 & 37 \\
900 & 22 & 39 \\
1,100 & 20 & 40 \\
1,300 & 20 & 41 \\
1,500 & 20 & 42 \\
\hline
\end{tabular}

\section{Principle of Efficiency Optimisation Based on DE Algorithm}

The DE algorithm is similar to the general evolutionary algorithm in structure, which consists of mutation, crossover and selection. First, the initial population with the satisfactory individual is randomly generated. Then, the vector difference of two arbitrary individuals is weighted. According to certain rules, the new individual is generated by summing the above vector difference and the third individual. The individuals with low adaptation degree will be eliminated by comparing new individuals and target individuals. The individuals with optimal value are finally obtained by continuous iterative operation. In this paper, the individual refers to the output current of each SRG. The optimal solution of current distribution is finally obtained through comparing the efficiency of parallel system under different distribution conditions.

\subsection{Process of efficiency optimisation}

Figure 4 shows the efficiency optimisation process based on DE algorithm. It can be found from Eq. (4) that there is a total of $M-1$ free variables in the problem of efficiency optimisation, including $I_{1}, I_{2}, \ldots, I_{M-1}$. The $M^{\text {th }}$ variable consists of the load current and M-1 free variables, as shown in Eq. (5).

$$
I_{M}=I_{L}-\sum I_{k} \quad(k=1,2, \ldots, M-1)
$$

Therefore, the number of load currents and parallel SRGs should be obtained first. Then, the corresponding initial population is generated according to the specified value of output current of each parallel SRG. According to certain rules, we adopt the mutation operation to randomly select three individuals and then utilise the crossover operation and the selection operation to generate the individual with high adaptation degree. Finally, the reference output currents of each SRG with optimal overall efficiency are generated by continuous iteration. 


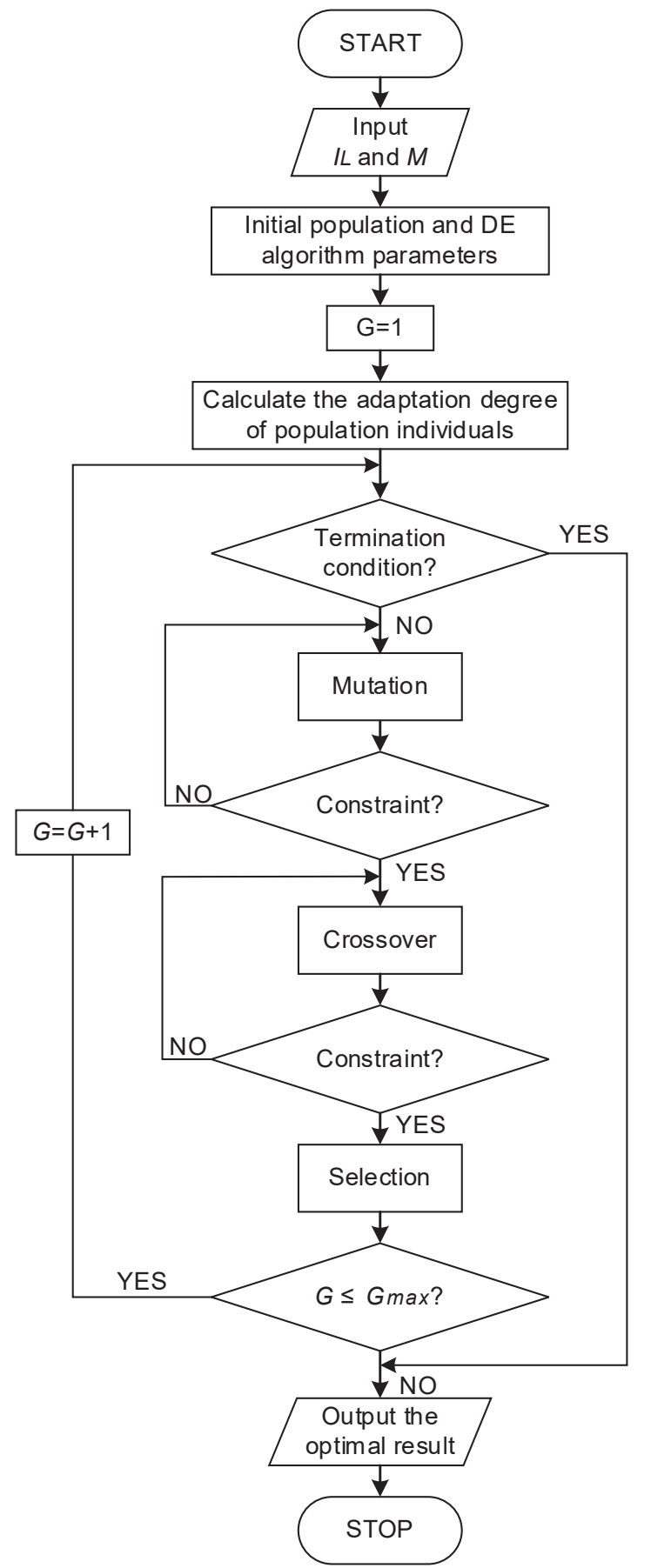

Fig. 4. Efficiency optimisation flow chart based on DE algorithm. DE, differential evolution.

In addition, Figure 4 also shows that we add the judgement after the mutation and crossover to make the individuals generated by the mutation and crossover satisfy the constraints. The next operation is not initiated until the constraints are met.

The selection and setting of key parameters play crucial roles in the output result and the convergence speed of the algorithm. The key parameters of algorithm mainly include mutation operator $(F)$, crossover factor $(C R)$ and group size $(S)$. 


\subsection{Basic operation of optimisation and parameter design}

a) Generation of initial population. The $S$ reflects the diversity of individuals in the population to a certain extent. The larger the $S$, the stronger the diversity of individuals. However, an excessive $S$ will lead to the high complexity of operation process, making the convergence time of algorithm longer. The low $S$ will lead to the difficulties in mutation operation. Therefore, we will find an appropriate $S$ to implement easily global convergence and find the optimal solution of efficiency. The number of individuals is generally the dimension of problem space of five to ten times. The basic operation of the generation of initial population in this paper is

$$
I_{i j}(0)=\operatorname{rand}_{i j}(0,1)\left(I_{i j}{ }^{M A X}-I_{i j}{ }^{M I N}\right)+I_{i j}{ }^{M I N}
$$

where $I_{i j}{ }^{\text {MAX }}$ and $I_{i j}{ }^{\text {IN }}$ are the maximum and minimum of output current respectively, $\operatorname{rand}_{i j}(0,1)$ is a random number in $[0,1]$.

b) Mutation operation. The basic mutation operation in this paper is

$$
h_{i j}(g)=I_{p 1 j}(g)+F\left(I_{p 2 j}(g)-I_{p 3 j}(g)\right) \quad F \in[0,2]
$$

where $I_{p 1 j}, I_{p 2 j}$ and $I_{p 3 j}$ refer to three individuals selected randomly from population. Their subscripts represent the ordinal numbers of individuals in the population, and $g$ represents the number of current iterations. $F$ represents mutation operator, which affects the diversity of individuals and the convergence of the algorithm.

To keep a certain convergence speed and prevent the algorithm from falling into local optimisation, the $F$ of linear change in this paper is

$$
F=\left(F_{M A X}-F_{M I N}\right) \frac{G-g}{G}+F_{M I N}=(1.2-0.3) \frac{G-g}{G}+0.3=0.9 \frac{G-g}{G}+0.3
$$

where $F_{\text {MAX }}$ and $F_{\text {MIN }}$ are the maximum and minimum of $F$, respectively, and $G$ is the total number of iterations. Equation (8) shows that the $F$ increases first and then decreases with the increase of the number of iterations, which is compatible with the algorithm convergence condition. The above method not only effectively reduces the convergence time but also maintains the diversity of individuals.

c) Crossover operation. The DE algorithm possesses the corresponding crossover operation, which is similar to chromosome genetic recombination in nature. Cross-combination of individuals in the population is beneficial in that it facilitates retention of the original population characteristics and enhances the diversity of individuals. Therefore, individuals generated by crossover operation will be experimental individuals of selection operation. The crossover operation in this paper is

$$
v_{i j}(g+1)=\left\{\begin{array}{l}
h_{i j}(g+1), \quad \operatorname{randl}_{i j} \leq C R \text { or } j=1, \ldots, D \\
I_{i j}(g), \text { randl }_{i j}>C R
\end{array} \quad C R \in[0,1]\right.
$$

where rand $_{i j}$ is a random number in $[0,1]$, and $C R$ is the crossover rate.

Equation (9) shows that the selection of $C R$ affects the actual participation of components of each individual, the population diversity and the approach of algorithm approximation. The low $C R$ will lead to the immature of algorithm, and on the other hand, the high $C R$ easily makes convergence speed slow. The $C R$ adopted in this paper is

$$
C R=\frac{C R_{M A X}-C R_{M I N}}{G} g+C R_{M I N}=\frac{(0.9-0.2)}{G} g+0.2=\frac{0.7}{G} g+0.2
$$

where $C R_{M A X}$ and $C R_{\text {MIN }}$ are the maximum and minimum of $C R$, respectively.

Equation (10) shows that the $C R$ reaches a very small value at the beginning of the iteration and thus transitions from a small to large value gradually. The $C R$ with a large value will effectively improve the convergence speed of the algorithm at the end of the iteration. Figure 5 shows the diagrammatic drawing of crossover operation. 


\begin{tabular}{|c|c|c|c|c|c|c|}
\hline$h i(g+1)$ & $h_{i 1}$ & $h_{i 2}$ & $\ldots$ & $h_{i j}$ & $\ldots$ & $h_{i D}$ \\
\hline $\operatorname{li}(g)$ & $l_{i 1}$ & $l_{i 2}$ & $\ldots$ & $l_{i j}$ & $\ldots$ & $l_{i D}$ \\
\hline rand $(l i j) \leq C R$ & TRUE & TRUE & $\ldots$ & FALSE & $\cdots$ & FALSE \\
\hline$j=1, \cdots, \mathrm{N}$ & FALSE & TRUE & $\ldots$ & TRUE & $\ldots$ & FALSE \\
\hline rand $(l i j) \leq C R$ & $h_{i 1}$ & $h_{i 2}$ & $\ldots$ & $h_{i j}$ & $\ldots$ & $l_{i D}$ \\
\hline
\end{tabular}

Fig. 5. The diagrammatic drawing of crossover operation of $D E$ algorithm. $C R$, crossover factor; $D E$, differential evolution.

d) Selection operation. To judge the advantages and disadvantages of the fresh individual generated by the above steps and the current individual, evaluation function can be used for selection. Consequently, the individuals with high adaptation degree will enter the next iteration. The overall efficiency of parallel power generation system is directly selected as the evaluation index in this paper. The specific operation is

$$
I_{i}(g+1)=\left\{\begin{array}{l}
v_{i}(g+1), \quad \eta\left(v_{i}(g+1)\right)>\eta\left(I_{i}(g+1)\right) \\
I_{i}(g), \quad \eta\left(v_{i}(g+1)\right) \leq \eta\left(I_{i}(g+1)\right)
\end{array}\right.
$$

\section{Simulation of Current Distribution Scheme}

On the basis of Eqs (3) and (4), it can be demonstrated that obtaining the efficiency characteristics of parallel SRGs is key to implementing the overall efficiency optimisation of parallel system. Therefore, the current distribution scheme with two parallel SRGs is simulated in this section. The rotation speed of Generator 1 is $500 \mathrm{r} / \mathrm{min}$; the $\theta_{\text {on }}$ is $22^{\circ}$; the $\theta_{\text {off }}$ is $35^{\circ}$. The rotation speed of Generator 2 is $1,300 \mathrm{r} / \mathrm{min}$; the $\theta_{\text {on }}$ is $20^{\circ}$; the $\theta_{\text {off }}$ is $41^{\circ}$, and the parallel load is $5 \Omega$.

To obtain the efficiency characteristics of two SRGs under different speeds, the data points are collected offline by simulation first. Then, the function relationship of each SRG is obtained by curve fitting. Figure 6 shows

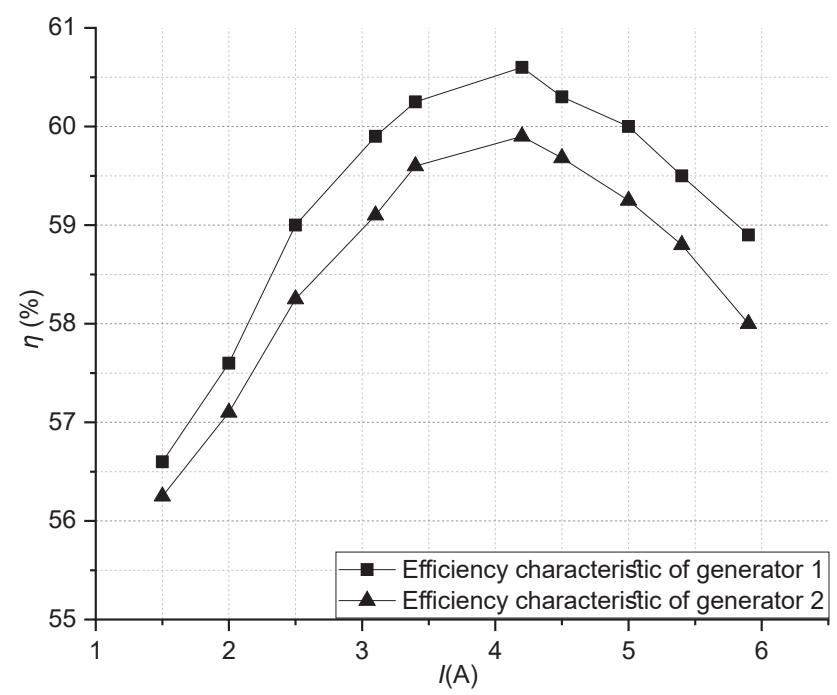

Fig. 6. Efficiency characteristic curve of generator by fitting. 
the efficiency characteristic curves of two SRGs after fitting. Finally, the polynomial with five order is used to fit the relevant data points and ensure a certain fitting accuracy.

Equation (12) and Table 2 show the efficiency characteristic expression and the coefficients under quintic polynomial.

$$
\eta_{k}=a_{5} I_{k}^{5}+a_{4} I_{k}^{4}+a_{3} I_{k}^{3}+a_{2} I_{k}^{2}+a_{1} I_{k}+a_{0} \quad(k=1,2)
$$

Equation (4) shows that $I_{1}$ is a free variable. The objective function of DE algorithm is

$$
\eta=\frac{I_{L}}{\frac{I_{1}}{\eta_{1}}+\frac{I_{L}-I_{1}}{\eta_{2}}}
$$

where the range of $I_{1}$ is $[1,6]$.

The output current $I_{2}$ presents the same constraints as $I_{1}$. Therefore, another constraint of $I_{1}$ is

$$
I_{L}-6<I_{1}<I_{L}-1
$$

To make $I_{1}$ satisfy the constraint, the load current $I_{L}$ under steady state should be calculated. First, the parallel power generation system keeps stable as a result of the maximum value CSC, to make $I_{L}$ constant. Based on the above, the efficiency of parallel power generation system is optimised by adjusting the reference current of each SRG. The current distribution control method based on efficiency optimisation is introduced to the simulation on the basis of the maximum value CSC, as shown in Figure 7. The different control method is chosen from judging the change of the load current. Figure 8 shows the output voltage and output current waveforms of two parallel SRGs at the beginning of parallel operation.

The parallel power generation system firstly utilises the CSC method at the 0.4 th $\mathrm{s}$, and then the output currents of two SRGs, which are about $2 \mathrm{~A}$, keep equilibrium at 0.5 th $\mathrm{s}$. The CSC method switches to EOC method during $0.08 \mathrm{~s}$, and then the parallel power generation system reaches equilibrium at about 0.7 th $\mathrm{s}$. Meanwhile, the output current of Generator 1 is about 3A, and the output current of Generator 2 is about $1 \mathrm{~A}$. Figure 8(b) shows that the output voltage of two parallel SRGs are essentially kept steady at voltage rating $20 \mathrm{~V}$ in the whole process,

Table 2. Efficiency characteristic coefficient

\begin{tabular}{lcccccc}
\hline & $a_{5}$ & $a_{4}$ & $a_{3}$ & $a_{2}$ & $a_{1}$ & $a_{0}$ \\
\hline \hline Generator 1 & 0.0048 & -0.05373 & 0.12043 & -0.08835 & 2.61606 & 52.64754 \\
Generator 2 & 0.00611 & -0.08237 & 0.33579 & -0.75247 & 3.26091 & 52.20921 \\
\hline
\end{tabular}

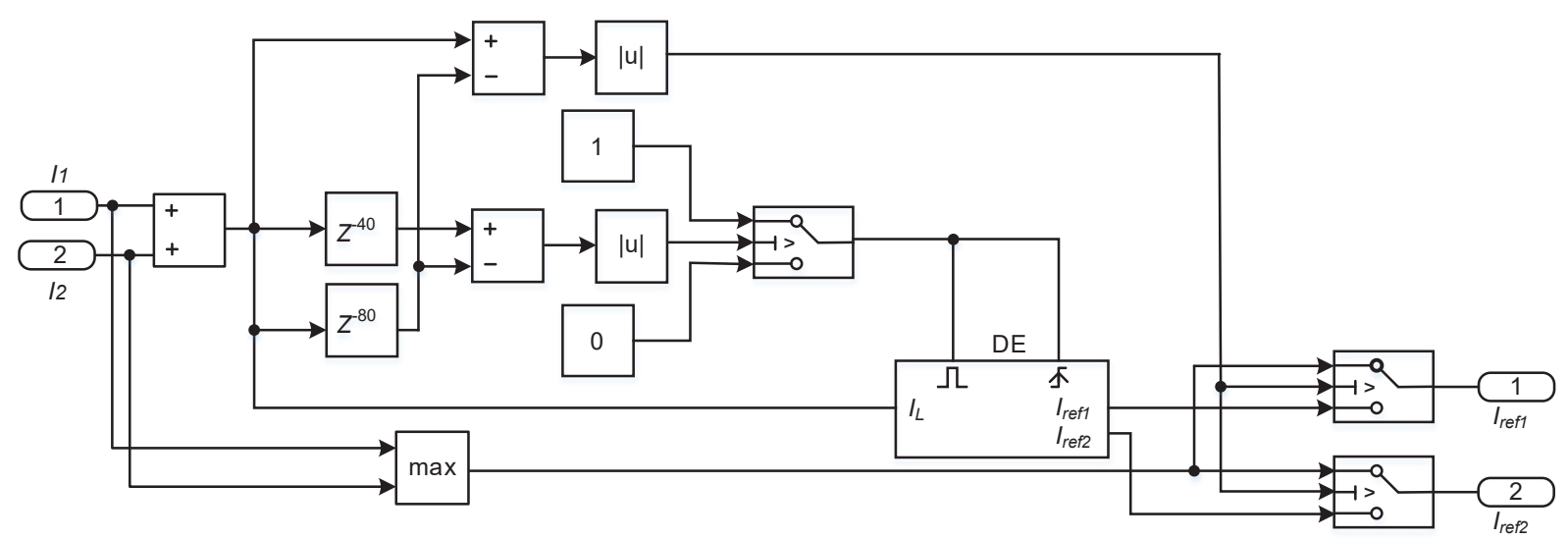

Fig. 7. Simulation model of current distribution control based on efficiency optimisation. 
except for transient fluctuation within control method switching. To verify the dynamic performance of the parallel system from various aspects, we simulate the sudden load-up and sudden load-reduction of parallel system, as shown in Figure 9.

Figure 9 shows that the parallel system keeps equilibrium under EOC method before the first second. After the first second, the load current changes as the load resistance changes from $5 \Omega$ to $3 \Omega$. The EOC method is switched automatically to CSC method due to the change of load current, and then the output current of each SRG is about 3.3 A after the above adjustment. The output voltages of each SRG decrease as sudden load-up and then returns to the rating during $0.03 \mathrm{~s}$. Finally, the CSC method is switched to the EOC method when the output current of each SRG reaches stability, and then the reference currents of each SRG are adjusted again.

The load resistance returns to $5 \Omega$ under sudden load-reduction at 1.8 th $\mathrm{s}$. In contrast with sudden load-up, the output voltage of each SRG increases briefly, and then the control method is switched to CSC method quickly. Finally, the parallel system keeps stable again during $0.1 \mathrm{~s}$, and then runs in EOC method again.

Therefore, the selection of control method depends on the changes of the load current. The control method can be flexibly switched between the CSC method and the EOC method in the above process. In addition, the small voltage fluctuation generated by switching of control method is still within the tolerance range.

Table 3 and Figure 10 show that the overall efficiency of parallel system increases with the increase of the load current under two-control method, and the overall efficiency of the system starts to decrease as the load current increases to about $8 \mathrm{~A}$. In addition, the overall efficiency under EOC method is always higher than CSC method.

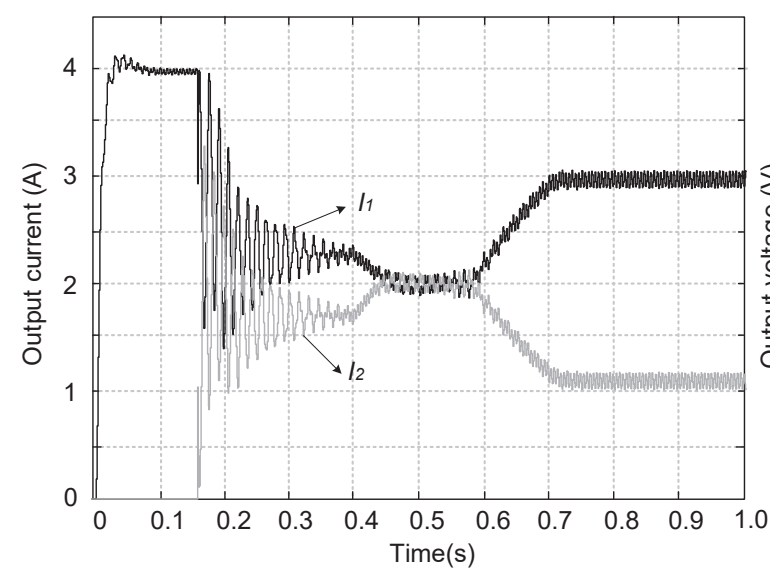

(a)Output current of each parallel generator

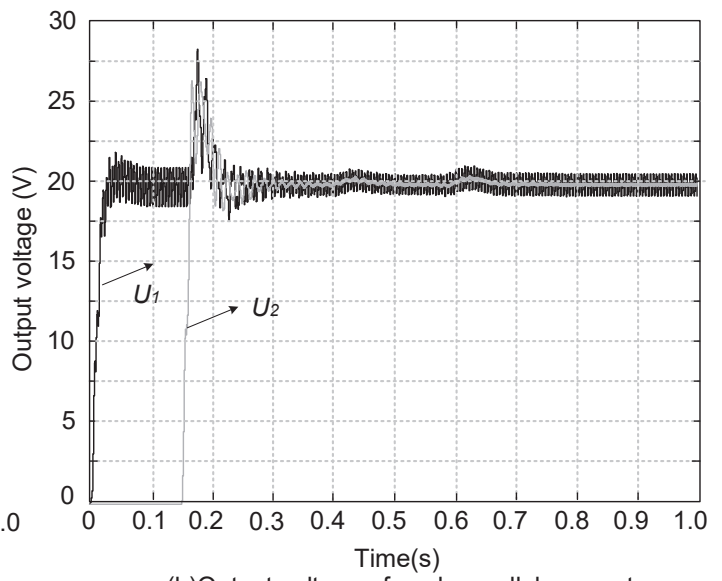

(b)Output voltage of each parallel generator

Fig. 8. The output current and output voltage waveforms at the beginning of parallel operation.

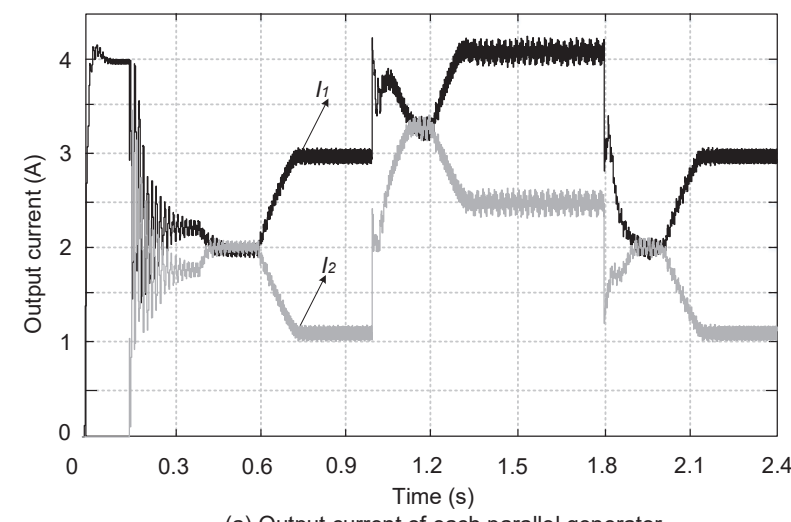

(a) Output current of each parallel generator

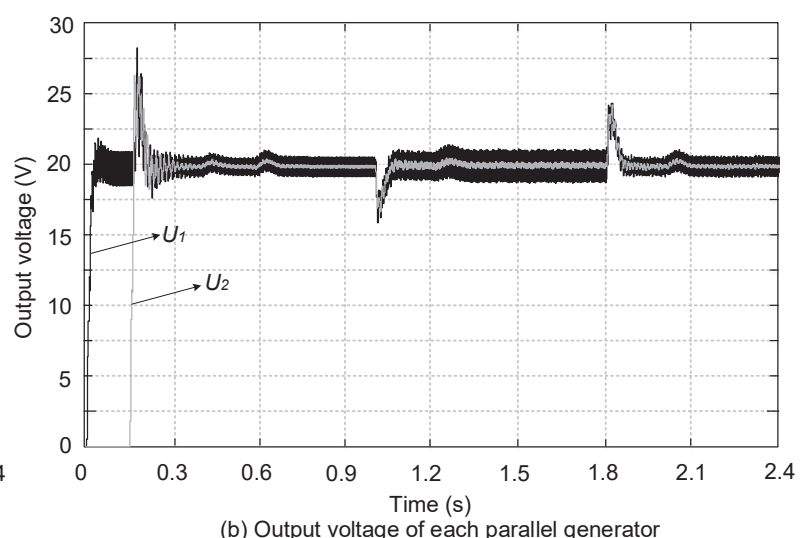

(b) Output voltage of each parallel generator

Fig. 9. The output current and voltage waveforms of each generator under sudden load-up and sudden load-reduction. 
Table 3. Current distribution of each parallel SRG under different load currents

\begin{tabular}{lcc}
\hline Load current $(\mathrm{A})$ & Reference current of Generator 1 (A) & Reference current of Generator 2 (A) \\
\hline \hline 3 & 2 & 1 \\
4 & 2.96 & 1.04 \\
5 & 3.87 & 1.13 \\
6 & 4.03 & 1.97 \\
7 & 4.25 & 2.75 \\
8 & 4.5 & 3.5 \\
9 & 4.94 & 4.06 \\
\hline
\end{tabular}

SRG, switched reluctance generator.

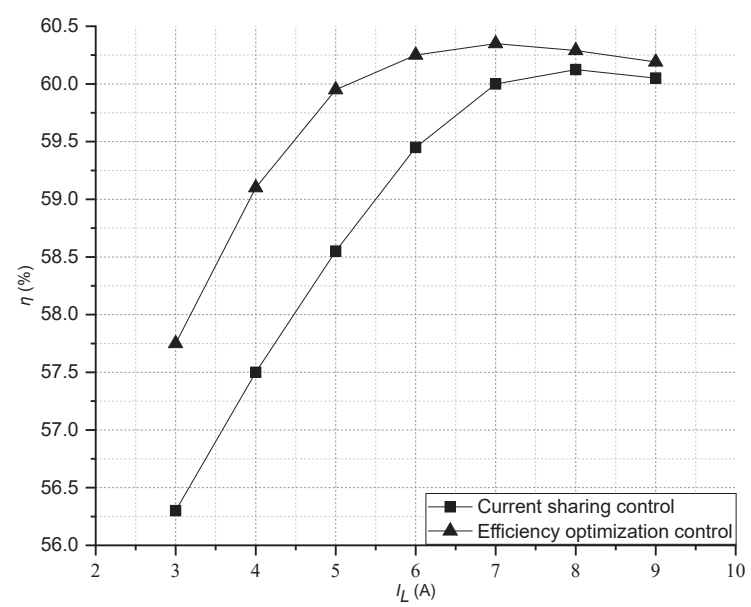

Fig. 10. The overall efficiency of parallel system under two methods

\section{Experiment of Current Distribution Scheme}

Figure 11 shows the output voltage and output current waveforms of parallel SRG before and after CSC method. The rotating speed of Generator 1 is $500 \mathrm{r} / \mathrm{min}$; the $\theta_{\text {on }}$ and the $\theta_{\text {off }}$ are $22^{\circ}$ and $35^{\circ}$, respectively. The rotating speed of Generator 2 is $1,200 \mathrm{r} / \mathrm{min}$; the $\theta_{\text {on }}$ and the $\theta_{\text {off }}$ are $20^{\circ}$ and $40^{\circ}$, respectively. The reference voltage of two SRGs
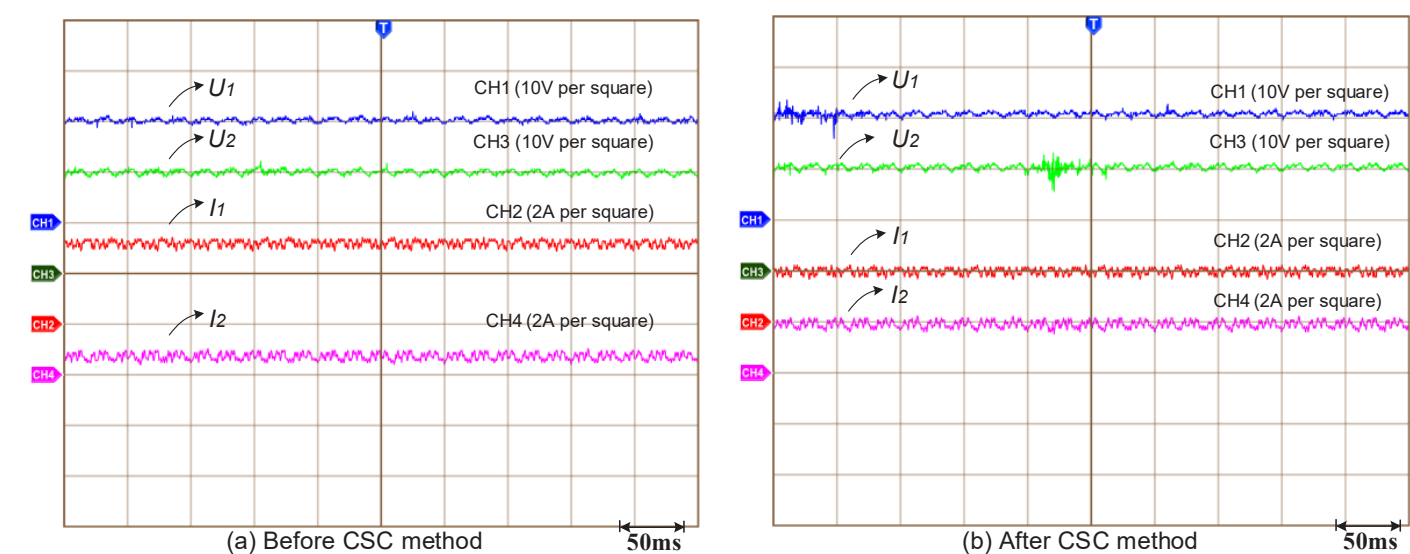

Fig. 11. The output voltage and current waveforms of each parallel SRG before and after CSC method. CSC, current sharing control; SRG, switched reluctance generator. 
is $20 \mathrm{~V}$. The load resistance takes $5 \Omega$. Figure $11(\mathrm{a})$ shows that the setting with different rotating speed results in the uneven output current of each parallel SRG. The output current of Generator 1 is $3.5 \mathrm{~A}$; the output current of Generator 2 is $0.5 \mathrm{~A}$. The output voltage of both SRGs is $20 \mathrm{~V}$. Figure 11 (b) shows that the output currents of each parallel SRG are kept basically even at 2 A under CSC method. In addition, the output voltage of each SRG rises to a certain extent.

Figure 12 shows the output voltage and output current of each parallel SRG under CSC method and variable load. The output voltage of each parallel SRG drops briefly under sudden load-up at first and then returns to the reference voltage after about $30 \mathrm{~ms}$. The output currents of each parallel SRG changes in the same way as the output voltages, which are $3 \mathrm{~A}$. Similarly, each parallel generator possesses current sharing well under sudden loadreduction in the whole process, except for transient voltage fluctuation.

Figure 13 shows the output voltage and output current of each parallel SRG under EOC method and variable load. The parallel power generation system runs under the CSC method first and then automatically switches to the EOC method when the system keeps stable.

In consequence, the parallel power generation system re-adjusts output current according to the changes of the load current to implement the efficiency optimisation. Each parallel SRG keeps stable in the whole operation, except for transient fluctuation during control method switching. Table 4 shows the overall efficiency of the system with different load currents under the CSC method and the EOC method.

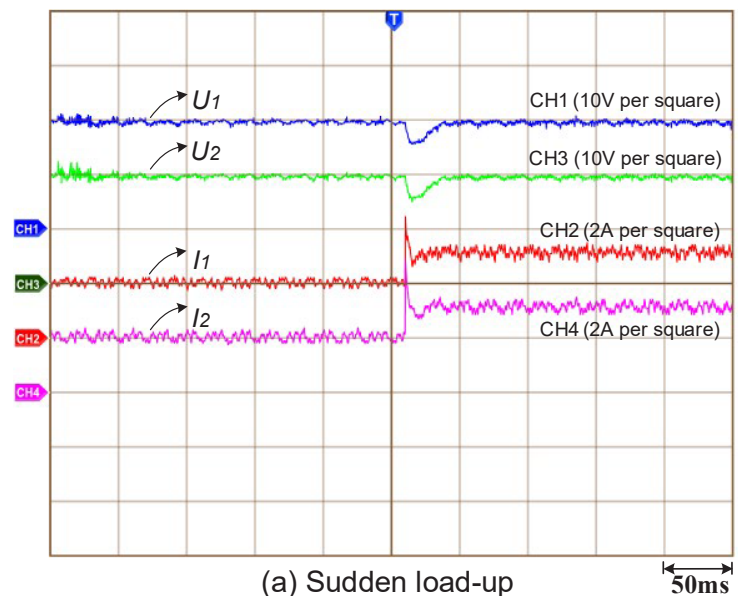

Fig. 12. The output voltage and current waveforms of each SRG under sudden load-up and load-reduction (CSC method). CSC, current sharing control; SRG, switched reluctance generator.

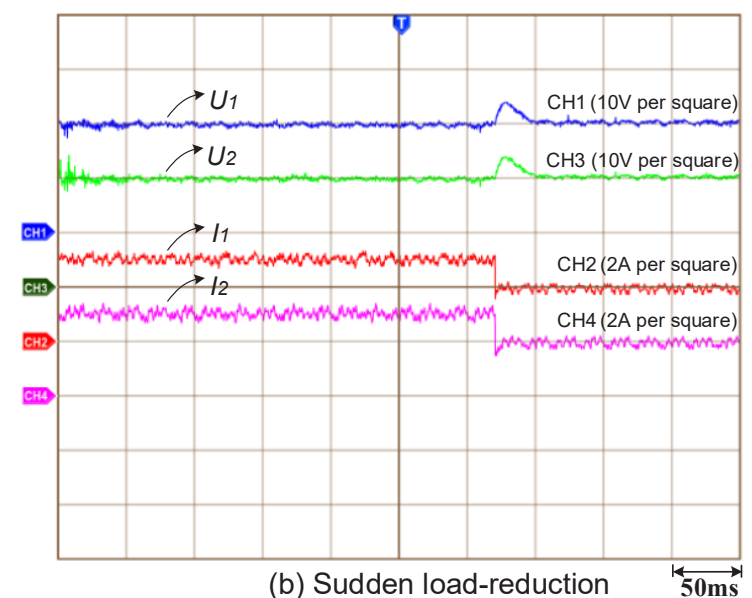

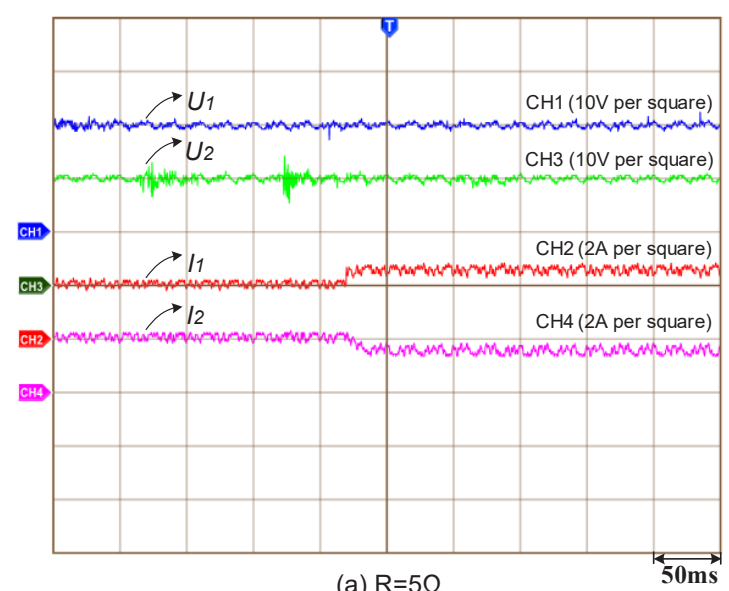

(a) $\mathrm{R}=5 \Omega$

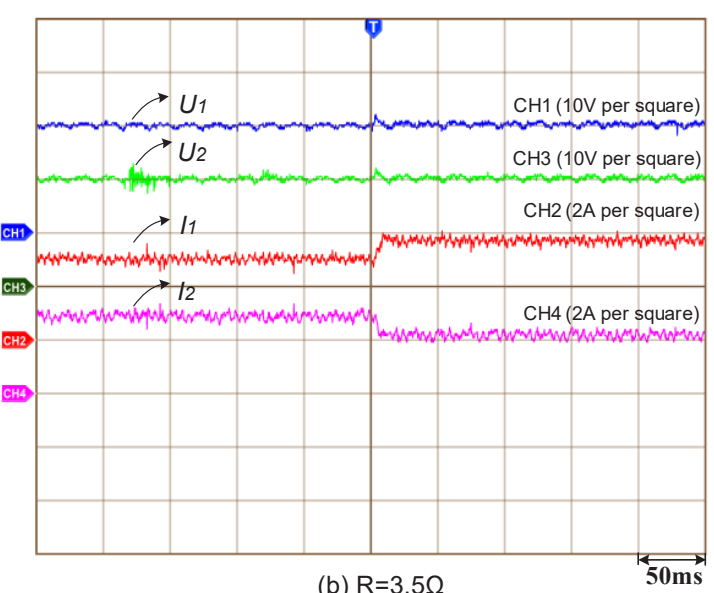

(b) $R=3.5 \Omega$

Fig. 13. The output voltage and current waveforms of each SRG under different load currents (EOC method). EOC, efficiency optimisation control; SRG, switched reluctance generator. 
Table 4. The comparison of overall efficiency under two control methods

\begin{tabular}{lcc}
\hline Load current $(A)$ & \multicolumn{2}{c}{ The overall efficiency of system (\%) } \\
\cline { 2 - 3 } & CSC method & EOC method \\
\hline \hline 3 & 56.1 & 57.3 \\
4 & 56.9 & 58.6 \\
5 & 57.6 & 59.2 \\
6 & 58.9 & 59.4 \\
7 & 59.2 & 60.3 \\
8 & 59.6 & 60.1 \\
\hline
\end{tabular}

CSC, current sharing control; EOC, efficiency optimisation control.

\section{Conclusion}

To promote the application of multi-machine parallel power generation system in the aerospace power supply system, a current distribution control method with efficiency optimisation is proposed in this paper. The EOC method and CSC method work together to optimise the overall efficiency of parallel system in the uneven current distribution control method. First, we obtain the efficiency expression of parallel power generation system on the basis of the efficiency characteristic curves of each SRG after fitting. Then, the optimal objective is confirmed as the overall efficiency of parallel system. Besides, the reference current of each parallel SRG is adjusted by utilising DE algorithm to transform the working point of each generator and implement the efficiency optimisation. In conclusion, the detailed simulation and experiment indicate that compared with the traditional CSC, the proposed method in this paper can effectively reduce unnecessary losses and enhance the overall efficiency of parallel power generation system.

\section{Acknowledgments}

This work is supported by Open Fund of State Key Laboratory of Operation and Control of Renewable Energy \& Storage Systems (China Electric Power Research Institute) (No. NYB51202001602).

\section{References}

Barros, T. A. D. S., Neto, P. J. D. S., Filho, P. S. N., Moreira, A. B. and Filho, E. R. (2017). An Approach for Switched Reluctance Generator in a Wind Generation System with a Wide Range of Operation Speed. IEEE Transactions on Power Electronics, 32(11), pp. 8277-8292.

Cai, J. and Deng, Z. Q. (2012). Sensorless Control of Switched Reluctance Motor Based on Phase Inductance Vectors. IEEE Transactions on Power Electronics, 27(7), pp. 3410-3423.

Chang, Y. C. and Liaw, C. M. (2008). On the Design of Power Circuit and Control Scheme for Switched Reluctance Generator. IEEE Transactions on Power Electronics, 23(1), pp. 445-454.

Chen, H. and Gu, J. J. (2010). Implementation of the Three-Phase Switched Reluctance Machine System for Motors and Generators. IEEE/ASME Transactions on Mechatronics, 15(3), pp. 421-432.
Chen, H., Sun, C. and Wang, Q. (2014). Analysis of FluxLinkage Characteristics of Switched Reluctance Linear Generator. IEEE Transactions on Applied Superconductivity, 24(3), pp. 1-5.

Chen, Z. X., Guo, Y. N., Chen, M. M. and Ge, L. S. (2016). Study on PI Sliding Mode Controller for Paralleled DC-DC Converter. In: Proceedings of the IEEE 8th International Power Electronics and Motion Control Conference (IPEMC-ECCE Asia), Hefei, 20-26 May 2016.

Choi, D., Byun, S. and Cho, Y. (2014). A Study on the Maximum Power Control Method of Switched Reluctance Generator for Wind Turbine. IEEE Transactions on Magnetics, 50(1), pp. 1-4.

Du, H., Jiang, C. and Wen, G. (2019). Current Sharing Control for Parallel DC-DC Buck Converters Based on Finite-Time Control Technique. IEEE Transactions on Industrial Informatics, 15(4), pp. 2186-2198. 
Gan, C., Wu, J., Hu, Y., Yang, S., Cao, W. and Guerrero, J. M. (2017). New Integrated Multilevel Converter for Switched Reluctance Motor Drives in Plug-in Hybrid Electric Vehicles with Flexible Energy Conversion. IEEE Transactions on Power Electronics, 32(5), pp. 3754-3766.

$\mathrm{Hu}$, Y. W., Huang, W. X. and Zhang, L. H. (2006). Research on Employing Starter/Generator System. Journal of Electrical Technology, 21(5), pp. 1-5.

Ju, R. R. (2008). Research on Aero DC StarterGenerator Control Unit. [online] Nanjing University of Aeronautics and Astronautics. Available from: www.cnki.net. [Accessed 5 January 2021].

Krishnamurthy, M., Edrington, C. S., Emadi, A., Asadi, P., Ehsani, M. and Fahimi, B. (2006). Making the Case for Applications of Switched Reluctance Motor Technology in Automotive Products. IEEE Transactions on Power Electronics, 21(3), pp. 659-675.

Li, J. Q. and Li, H. M. (2002). Summary on Development of Switched Reluctance Machine. Journal of North China Electric Power University, 29(1), pp. 1-5.

Liu, C., Liu, D. J., Zhu, X. Z. and Gao, Z. L. (2002). Study on Strategy of Switched Reluctance Generator without Position Sensor. China Journal of Electrical Engineering, 22(6), pp. 72-75.

Oyama, J., Higuchi, T. and Abe, N. (2010). The principle and Fundamental Characteristics of ACExcited Brushless Synchronous Motor. Electrical Engineering in Japan, 107(6), pp. 98-106.

Panov, Y. and Jovanovic, M. M. (2008). Loop Gain Measurement of Paralleled DC-DC Converters with Average-Current-Sharing Control. IEEE Transactions on Power Electronics, 23(6), pp. 2942-2948.

Peng, H., Yi, L., Deng, W. and Zhu, J. (2011). Increasing Output Power of Switched Reluctance Generator with Three-Level Power Converter. In: Proceedings of Asia-Pacific Power and Energy Engineering Conference, Wuhan, China, 25-28 March 2011.

Rahmanian, E., Akbari, H. and Sheisi, G. H. (2017). Maximum Power Point Tracking in Grid Connected Wind Plant by Using Intelligent Controller and Switched Reluctance Generator. IEEE Transactions on Sustainable Energy, 8(3), pp. 1313-1320.

Ren, X. Y., Wang, Y. K. and Chen, Y. (2019). Parallel Current Sharing Control of LLC Resonant
Converter Based on Virtual Impedance. Journal of Electrical Technology, 34(21), pp. 4540-4550.

Schofield, N. and Long, S. (2009). Generator Operation of a Switched Reluctance Starter/Generator at Extended Speeds. IEEE Transactions on Vehicular Technology, 58(1), pp. 48-56.

Sikder, C., Husain, I. and Sozer, Y. (2014). Switched Reluctance Generator Control for Optimal Power Generation with Current Regulation. IEEE Transactions on Industry Applications, 50(1), pp. 307-316.

Song, S. J., Ge, L. F., Liu, H. C. and Liu, W. G. (2014). Design and Multi-Objective Optimization Method of Switched Reluctance Machines. Journal of Electrical Technology, 29(5), pp. 197-204.

Sun, Z. G., Cheung, N. C., Zhao, S. W., Lu, Y. and Shi, Z. H. (2011). Design and Simulation of a Linear Switched Reluctance Generator for Wave Energy Conversion. In: Proceedings of the 4th International Conference on Power Electronics Systems and Applications, Hong Kong, China, 8-10 June 2011.

Torrey, D. A. (2002). Switched Reluctance Generators and Their Control. IEEE Transactions on Industrial Electronics, 49(1), pp. 3-14.

Xiong, L. X., Gao, H. L. and Xu, B. Y. (2009). Control Principle of Switched Reluctance Generator for Maximum Output Power. Journal of Motors and Controls, 13(2), pp. 250-254.

Zhang, H. and Pan, Z. P. (2004). Theoretic Analysis of Excitation Strategy and Simulation of Switched Reluctance Generators. Journal of Solar Energy, 25(2), pp. 162-170.

Zhang, T. J., Wei, J. D., Liu, P., Tao, W. J. and Zhou, B. (2018). An Integrated Motor-Drive and BatteryCharging System Based on Split-Field-Winding Doubly Salient Electromagnetic Machine. IEEE Transactions on Magnetics, 54(11), pp. 1-6.

Zhou, H., Wang, Y., Li, M. H., Li, K. P. and Lei, W. J. (2019). Analysis and Optimal Control of Transient Active Power Sharing between Islanded Parallel Virtual Synchronous Generators. Journal of Electrical Technology, 34(S2), pp. 654-663.

Zhou, Y. (2011). Research on the Digital Control Technology of Parallel DC Generators Based on CAN Bus. [online] Nanjing University of Aeronautics and Astronautics. Available from: www.cnki.net. [Accessed 10 January 2021] 